\title{
Highly sensitive MLH1 methylation analysis in blood identifies a cancer patient with low-level mosaic MLH1 epimutation
}

Estela Dámaso ${ }^{1 \dagger}$, Júlia Canet-Hermida ${ }^{1 \dagger}$, Gardenia Vargas-Parra ${ }^{1,2}$, Àngela Velasco ${ }^{2,3}$, Fátima Marín 1,2, Esther Darder ${ }^{3}$, Jesús del Valle ${ }^{1,2}$, Anna Fernández ${ }^{1}$, Àngel Izquierdo ${ }^{3}$, Gemma Mateu $^{4}$, Glòria Oliveras $^{4}$, Carmen Escribano ${ }^{5}$, Virgínia Piñol ${ }^{6}$, Hugo-lkuo Uchima ${ }^{6}$, José Luis Soto ${ }^{7}$, Megan Hitchins ${ }^{8}$, Ramon Farrés ${ }^{2}$, Conxi Lázaro ${ }^{1,9}$, Bernat Queralt ${ }^{10}$, Joan Brunet ${ }^{1,3,9,11}$, Gabriel Capellá ${ }^{1, *^{*}}$ (i) and Marta Pineda ${ }^{1, *^{*}+}$

\begin{abstract}
Constitutional MLH1 methylation (epimutation) is a rare cause of Lynch syndrome. Low-level methylation ( $\leq 10 \%)$ has occasionally been described. This study aimed to identify low-level constitutional MLH1 epimutations and determine its causal role in patients with $M L H 1$-hypermethylated colorectal cancer.

Eighteen patients with $M L H 1$-hypermethylated colorectal tumors in whom MLH1 methylation was previously undetected in blood by methylation-specific multiplex ligation-dependent probe amplification (MS-MLPA) were screened for MLH1 methylation using highly sensitive MS-melting curve analysis (MS-MCA). Constitutional methylation was characterized by different approaches.

MS-MCA identified one patient (5.6\%) with low-level MLH1 methylation ( 1\%) in blood and other normal tissues, which was confirmed by clonal bisulfite sequencing in blood. The patient had developed three clonally related gastrointestinal MLH1methylated tumor lesions at 22, 24, and 25 years of age. The methylated region in normal tissues overlapped with that reported for other carriers of constitutional MLH1 epimutations. Low-level MLH1 methylation and reduced allelic expression were linked to the same genetic haplotype, whereas the opposite allele was lost in patient's tumors. Mutation screening of $\mathrm{MLH1}$ and other hereditary cancer genes was negative.

Herein, a highly sensitive MS-MCA-based approach has demonstrated its utility for the identification of lowlevel constitutional MLH1 epigenetic mosaicism. The eventual identification and characterization of additional cases will be critical to ascertain the cancer risks associated with constitutional MLH1 epigenetic mosaicism.
\end{abstract}

Keywords: Constitutional MLH1 epimutation, Lynch syndrome, Methylation, Epigenetic mosaicism, Highly sensitive methodologies

\footnotetext{
*Correspondence: gcapella@iconcologia.net; mpineda@iconcologia.net †Estela Dámaso and Júlia Canet-Hermida contributed equally to this work and share first authorship.

${ }^{\dagger}$ Gabriel Capellá and Marta Pineda contributed equally to this work and share senior authorship.

${ }^{1}$ Hereditary Cancer Program, Catalan Institute of Oncology, Institut d'Investigació Biomèdica de Bellvitge (IDIBELL), ONCOBELL Program, Av. Gran Via de l'Hospitalet, 199-203, 08908 L' Hospitalet de Llobregat, Barcelona,

Spain

Full list of author information is available at the end of the article
}

(c) The Author(s). 2019 Open Access This article is distributed under the terms of the Creative Commons Attribution 4.0 International License (http://creativecommons.org/licenses/by/4.0/), which permits unrestricted use, distribution, and reproduction in any medium, provided you give appropriate credit to the original author(s) and the source, provide a link to the Creative Commons license, and indicate if changes were made. The Creative Commons Public Domain Dedication waiver (http://creativecommons.org/publicdomain/zero/1.0/) applies to the data made available in this article, unless otherwise stated. 


\section{Introduction}

Recent findings have indicated that underlying epimutations of certain genes in the normal tissue are associated with an elevated risk of particular tumor types [1-5]. This indicates that epigenetic events, apart from genetic alterations, maybe the initial step in the carcinogenesis process for these tumors [6]. When epimutations predisposing to disease are widely distributed across normal tissues, they are called constitutional epimutations.

Lynch syndrome (LS) is characterized by an increased risk for colorectal cancer (CRC) as well as other cancers (stomach, small intestine, and endometrium among others) [7]. It is mainly caused by germline genetic mutations in a mismatch repair (MMR) gene (MLH1, MSH2, $M S H 6$, or PSM2). In a small proportion of patients, LS is caused by a $M L H 1$ constitutional epimutation, in which monoallelic hypermethylation of the promoter $\mathrm{CpG}$ island throughout normal tissues is linked to a constitutional allele-specific silencing [5].

Ninety-seven index cases with a constitutional MLH1 epimutation have been reported so far [8-12]. Most are considered primary, arising apparently de novo and reversible between generations, whereas secondary epimutations are associated with a genetic variant in cis. Recently, we demonstrated that EPM2AIP1-MLH1 CpG island is the sole differentially methylated region in primary MLH1 epimutation carriers [9]. Available evidence suggests that constitutional epimutations cause a severe LS phenotype, including early-onset and multiple primary tumors [5].

The level of constitutional methylation varies among MLH1 epimutation carriers. Although the majority of cases identified to date have shown hemiallelic MLH1 methylation in blood, variable levels of methylation have frequently been reported (reviewed in [9]), including seven cases harboring low methylation levels $(\leq 10 \%)$ [13-16]. Nevertheless, low-level MLH1 methylation in blood has also been reported in healthy controls, confounding its interpretation [17]. Thus, the use of robust and sensitive approaches is critical to determine the true prevalence of constitutional epimutations and to ascertain a putative role of mosaic $M L H 1$ epimutation in cancer predisposition.

The main aim of this study was to identify patients with low levels of constitutional epigenetic mosaicism of the MLH1 gene using highly sensitive methylation analysis techniques and explore its role in cancer predisposition.

\section{Patients and methods}

\section{Patients and samples}

Patients were identified through the Cancer Genetic Counseling Units at the Catalan Institute of Oncology from 1998 to 2016. Eighteen individuals presenting with MLH1-methylated CRC before 50 years of age, or multiple tumors before 60 years, were studied (Additional file 1: Figure S1; Additional file 2: Table S1) after excluding two previously reported bona fide constitutional $M L H 1$ epimutations [18]. Their levels of $M L H 1$ methylation in PBL (peripheral blood leukocytes) previously assessed by MSMLPA were between 0 and $4 \%$ at the Deng $C$ and D regions of the $M L H 1$ promoter CpG island, hence were below the limit of detection by this technique (10\%) and considered negative (Additional file 2: Table S1) [19]. Twenty case-matched healthy individuals (matched by age, race, and geographic location) were included as controls. In addition, 61 LS cases harboring MMR genetic mutations, 12 constitutional MLH1 epimutation carriers, and 41 healthy controls were included as reference groups for comparative global methylome analyses [9]. Written informed consent was obtained from all individuals, and the ethics committee of the respective hospitals approved the study. Sample processing is detailed in Additional file 3: Supplementary Methods.

\section{Methylation testing}

The levels of methylation at the $M L H 1$ promoter in biological samples were assessed by several methods (Additional file 3: Supplementary Methods and Additional file 12: Table S5): (i) Methylation-specific multiplex ligation-dependent probe amplification (MS-MLPA) using the SALSA MLPA ME011 Mismatch Repair genes probemix (MRC-Holland). (ii) Methylation-specific melting curve analysis (MS-MCA): bisulfite-treated DNA was amplified within the Deng $C$ and D regions in a nested PCR on a LightCycler 480 II; the analytical sensitivity of MS-MCA was assessed using serial dilutions $(100,75,50,25,10,5,4$, 3,2 , and $1 \%$ ) of the RKO cell line (biallelic $M L H 1$ methylation; $100 \%$ methylated) into unmethylated ( $0 \%$ methylated) Whole Genome Amplification (WGA) DNA. The analytical sensitivity was $1 \%$ and $10 \%$ for the $C$ and $D$ regions, respectively (Additional file 4: Figure S2A-B). (iii) Pyrosequencing: bisulfite-treated DNA was amplified within the Deng $\mathrm{C}$ and intron 1 regions with biotin-labeled primers; the estimated analytical sensitivity was $4 \%$ and $5 \%$ at Cregion and intron 1, respectively (Additional file 5: Figure S3A-B). (iv) Clonal bisulfite sequencing of fragments of the MLH1 promoter encompassing the c.-93G $>\mathrm{A}$ promoter SNP was performed to confirm the low-level methylation detected by MS-MCA. (v) Finally, genome-wide methylation profiling was performed using Infinium Human Methylation 450K Beadchip, as previously described [9].

\section{MLH1 expression and loss of heterozygosity analyses}

Human lymphocytes from case 29 were cultured in PBMAX Karyotyping Medium (Life Technologies, Carlsbad, CA) in the absence and presence of puromycin (Sigma, St. Louis, MO). Puromycin was used to prevent potential degradation of unstable transcripts by the 
nonsense-mediated decay mechanism. The impact of MLH1 promoter methylation on allelic expression was assessed by measuring the relative levels of the two MLH1 alleles at exonic SNP c.655A>G (rs1799977) in cDNA/gDNA by single-nucleotide primer extension (SNuPE), as previously described [18].

Loss of heterozygosity ( $\mathrm{LOH}$ ) was assessed by SNUPE as the ratio of tumor-DNA/distal normal-DNA at c.655A $>$ G. Clonal sequencing of $M L H 1$ cDNA was performed to determine the phase between the heterozygous c.655A $>\mathrm{G}$ (exonic) and c.-93G $>\mathrm{A}$ (promoter) variants.

\section{Germline mutational analysis}

Hereditary cancer genes (including $M L H 1$ gene) were screened for rare and mosaic germline variants, using analytical pipelines to detect mosaicism, as described in Additional file 3: Supplementary Methods.

\section{Immunohistochemical staining analysis}

Formalin-fixed, paraffin-embedded tissue sections representative of the tumors were studied for CK7, CK20, CDX2, MUC1, MUC2, and MUC5 protein expression using standard immunohistochemistry techniques (see Additional file 3: Supplementary Methods).

\section{Somatic mutational analyses}

Mutations in KRAS, NRAS, and BRAF were analyzed in tumors with the Idylla ${ }^{\mathrm{Tw}}$ platform (Biocartis, Mechelen, Belgium). FFPE tumor tissue sections were placed directly into the Idylla system cartridge according to the manufacturer's instructions (Idylla ${ }^{\text {тм }}$ KRAS Mutation Test and NRAS-BRAF Mutation Test) and were analyzed for mutations in codons $12,13,59,61,117$, and 146 of $K R A S$; in codons $12,13,59,61,117$, and 146 of $N R A S$; and in codon 600 of BRAF.

Tumor samples were further analyzed using the NGS customized panel of 126 genes (I2HCP v2.1) as described above. To determine somatic variants, germline variants identified in the paired blood with a variant allele frequency $(\mathrm{VAF})>0.1$ were subtracted from tumor variants. Variant calls with lower than $30 \mathrm{x}$ coverage, $\mathrm{VAF}<0.05$, or out of the region of interest (gene exons \pm $20 \mathrm{bp}$ ) were excluded. Somatic variants with VAF $>0.2$ in at least one tumor location were considered. The clonal relatedness between pairs of tumor samples based on their mutational profiles was tested using the SNVtest of the Clonality R package, which evaluates evidence for clonality against null hypothesis that the two tumors are independent [20]. Reference frequencies of somatic mutations were obtained from TCGA CRC MSI-H cohort with mutational data available $(n=28)$ [21]. For mutations not previously observed in the TCGA cohort, the reference frequency was set to 0.033
(1/29, being 29 the sum of the TCGA CRC MSI-H cases plus our patient).

\section{Results}

Highly sensitive $M L H 1$ methylation screening

The MS-MCA pattern of MLH1 promoter (region C) of PBL from DNA healthy controls was the same as the unmethylated WGA sample, indicating the absence of detectable methylation in the control group (Additional file 4: Figure S2C). Likewise, 17 out of 18 patients harboring MLH1-hypermethylated CRC shared a nonmethylated pattern. In contrast, PBL from case 29 showed the presence of methylation at a level of around 1\% (Fig. 1a and Additional file 4: Figure S2D). After confirmation of the same low methylation levels in an independently extracted blood sample (Fig. 1a), we decided to study the case in depth.

\section{Clinicopathological characterization}

Patient 29 is a woman who consecutively presented three MLH1-methylated gastrointestinal tumor lesions described as a low-grade (moderately differentiated) colorectal adenocarcinoma (pT4N1) at age 22, a welldifferentiated small bowel adenocarcinoma (pT4N1) at age 24, and a well-differentiated tubular adenocarcinoma of the stomach (pT2N1) at 25 years of age (Fig. 1b). According to the clinical presentation and macroscopic description, the three lesions were treated as primary independent tumors. The patient has no disease recurrence after 9 years of follow-up. No family history of cancer was reported among her first-degree relatives (Fig. 1b).

Of note, the three tumor lesions shared the same immunohistochemical staining pattern, showing negative expression of CK7 and CK20 markers and positive expression of CDX2, MUC1, MUC2, and MUC3 (Additional file 6: Figure S4). Also, 56\% (51 out of 91) of the somatic variants identified in these lesions were shared between them, strongly suggesting a common origin $(p<0.001$, clonality package) (Fig. 2a, b). The shared variants included mutations in key genes of intestinal carcinogenesis (e.g., APC K562fs, V782fs, and Q205X), well-known cancer driver mutations (KRAS G12D), and recurrent homopolymer deletions characteristic of MSI tumors (e.g., TGFBR2 K153fs).

\section{Confirmation of the mosaic constitutional MLH1 epimutation}

The presence of $M L H 1$ methylation was confirmed by MS-MCA in all embryonic layers since methylation was detected in endodermal (gastric, small bowel, and colon mucosa) and ectodermal tissues (oral mucosa and skin fibroblasts) at similar levels than in PBL (mesoderm) (Additional file 4: Figure S2). In addition, slightly higher levels of methylation were also 


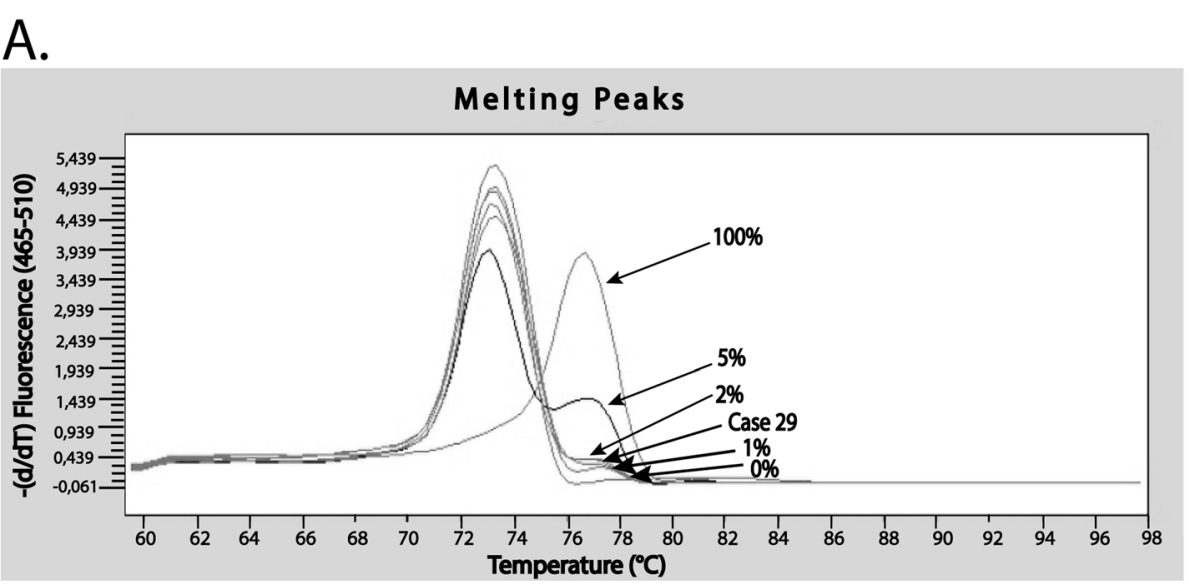

B.

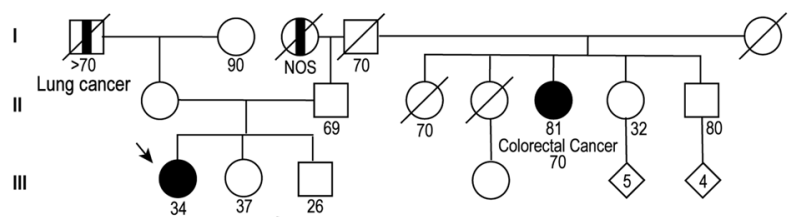
Colorectal cancer 22 ] MSI+ $\begin{array}{ll}\text { Small bowel cancer } 24 & \text { MLH1/PMS2- } \\ \text { Gastric cancer 25 } & \text { MLH1 met }\end{array}$

C.

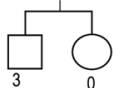

Sequence: GAGYGGATAGYGATTTTTAAYGYGTAAGYGTA
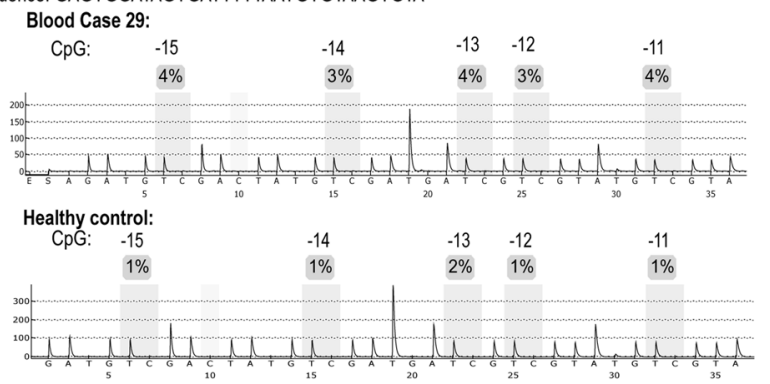

D.

rs 1800734

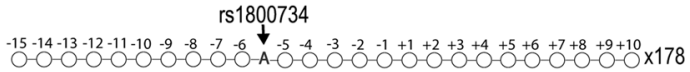

$0-0-0-0-0-0-0-0,0-0-0-0-0-0-0-0-0-0-0-0-0-0 \times 175$

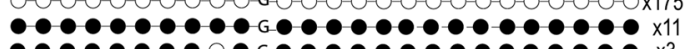

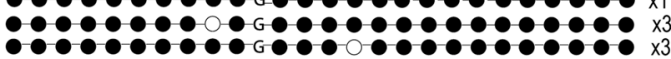

$\because: \bullet: \bullet: \bullet: \bullet: G \bullet: \bullet: \bullet: \bullet: \bullet: \bullet: \bullet: \mathrm{x}^{3}$

Fig. 1 Identification and characterization of constitutional MLH1 epigenetic mosaicism in case 29. a Methylation analysis by MS-MCA of the MLH1 promoter C-region in blood DNA from case 29. The patient shows levels of methylation around 1\%. b Family pedigree from case 29. The epimutation carrier is indicated by an arrow. Circles, females; squares, males; filled, cancer affected; vertical line at center, not otherwise specified. Cancer location and age at diagnosis are indicated. Generations are indicated on the left margin in Roman numerals. c Methylation analysis by pyosequencing of the MLH1 promoter C-region in blood DNA from case 29 and one healthy control. Each CpG is numbered according its position relative to the translation initiation codon. $\mathbf{d}$ Clonal bisulfite sequencing of the MLH1 promoter in PBL DNA from case 29. Each horizontal line represents a specific allele. CpG dinucleotides are depicted by circles. Black and white circles indicate methylated and unmethylated CpG sites, respectively. The allele at rs1800734 (c.-93G>A) is indicated as A or G. Methylation in case 29 is confined to the G allele. Each CpG analyzed is numbered according to its position relative to the translation initiation codon

detected in normal tissue samples by pyrosequencing, but below the analytical sensitivity threshold of this technique (Fig. 1c; Additional file 5: Figure S3). In contrast, previous MS-MLPA analyses had reported only background methylation levels in blood DNA and in normal gastrointestinal tissues $(\leq 10 \%)$ (Additional file 7: Table S2).

Considering the previous observations, MLH1 methylation in blood was further assessed by clonal bisulfite sequencing (Fig. 1d) to confirm the presence and density 


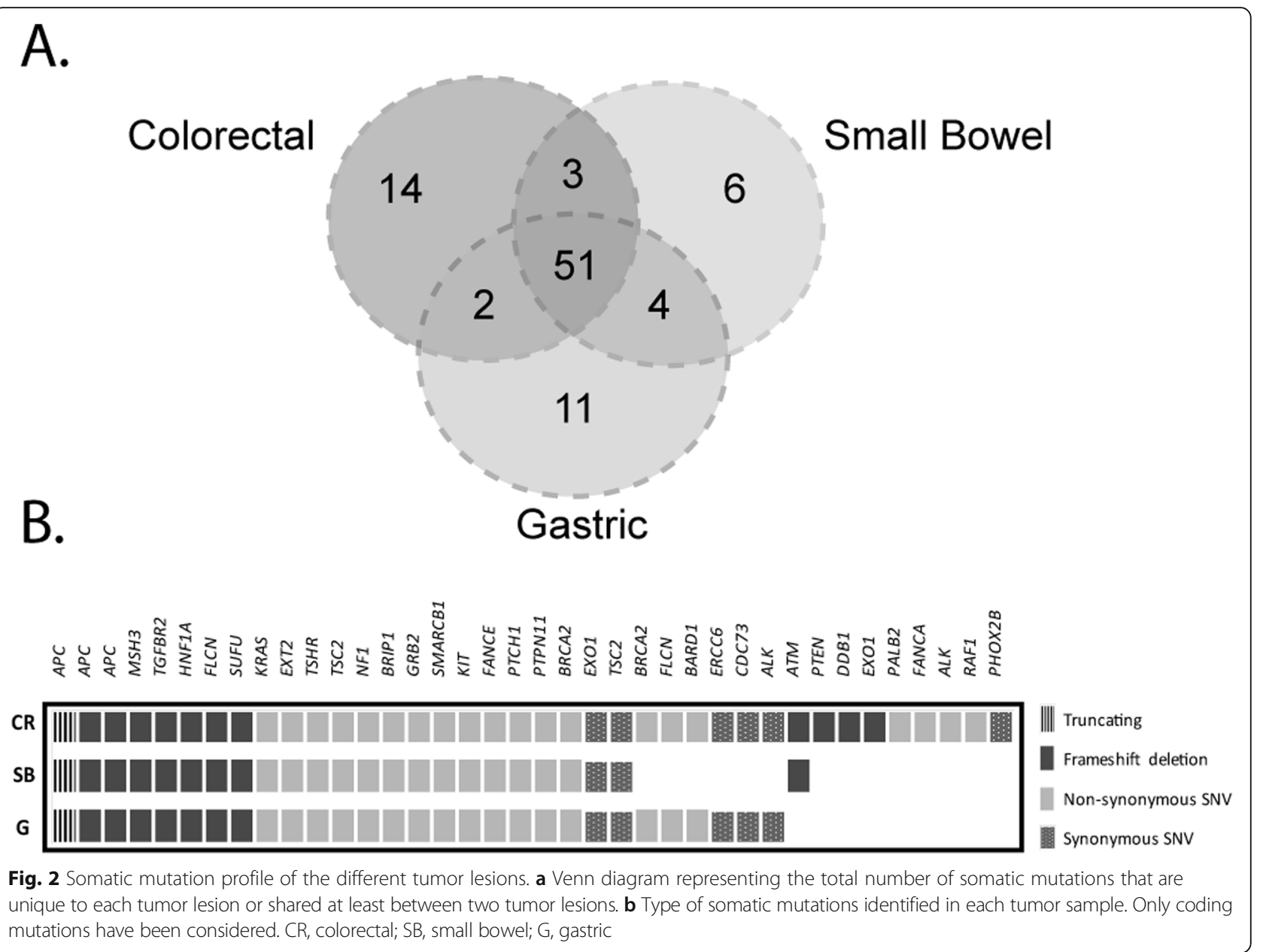

of methylation in individual alleles. Nineteen methylated clones were identified out of 372 analyzed (5\% methylated alleles; 95\% CI 0.01-0.05), all of them displaying dense monoallelic methylation linked to the $G$ allele at c.-93G >A (rs1800734), for which patient 29 was heterozygous. Of note, in cis genetic variants on the methylated $\mathrm{G}$ alleles were not detected in the region analyzed, including c.-27C $>\mathrm{A}$ (which has previously been linked to secondary $M L H 1$ epimutations) [22]. In all, clonal bisulfite sequencing supported the robustness of the MSMCA results.

\section{Characterization and classification of $M L H 1$ epimutation}

Sequencing of $M L H 1$ cDNA clones showed that the mosaic methylation of the c.-93G allele was in phase with the c.655G allele in patient 29 (Fig. 3a). Consistent with the low percentage of methylation associated with the c.-93G/c.655G allele, a slight reduction in the expression of this allele was observed in MLH1 transcripts, as compared to the $M L H 1$ expression in two control individuals (Fig. 3b). Accordingly, the three tumors showed somatic LOH on the opposite allele (c.655A) (Fig. 3c).
Global methylome array analysis revealed slightly higher levels of methylation across the MLH1 promoter in blood and normal colonic mucosa samples from patient 29 compared to controls (Fig. 4a, b), encompassing the same region previously shown to be differentially methylated in carriers of a constitutional MLH1 epimutation [9].

Of note, germline pathogenic variants were not found in the coding regions of hereditary cancer genes including $M L H 1$ (its promoter sequence was also analyzed) in patient 29 (Additional file 8: Table S3). Furthermore, no MLH1 germline copy number alterations were identified (Additional file 9: Figure S5). These findings, coupled with the lack of a cancer family history, suggest that patient 29 is the carrier of a primary epimutation.

\section{Discussion}

We report the finding of low-level constitutional MLH1 epigenetic mosaicism in a woman who suffered from three sequential MLH1-methylated tumor lesions of the upper abdominal area in her early twenties. A comprehensive analytical approach that combined highly sensitive MS- 

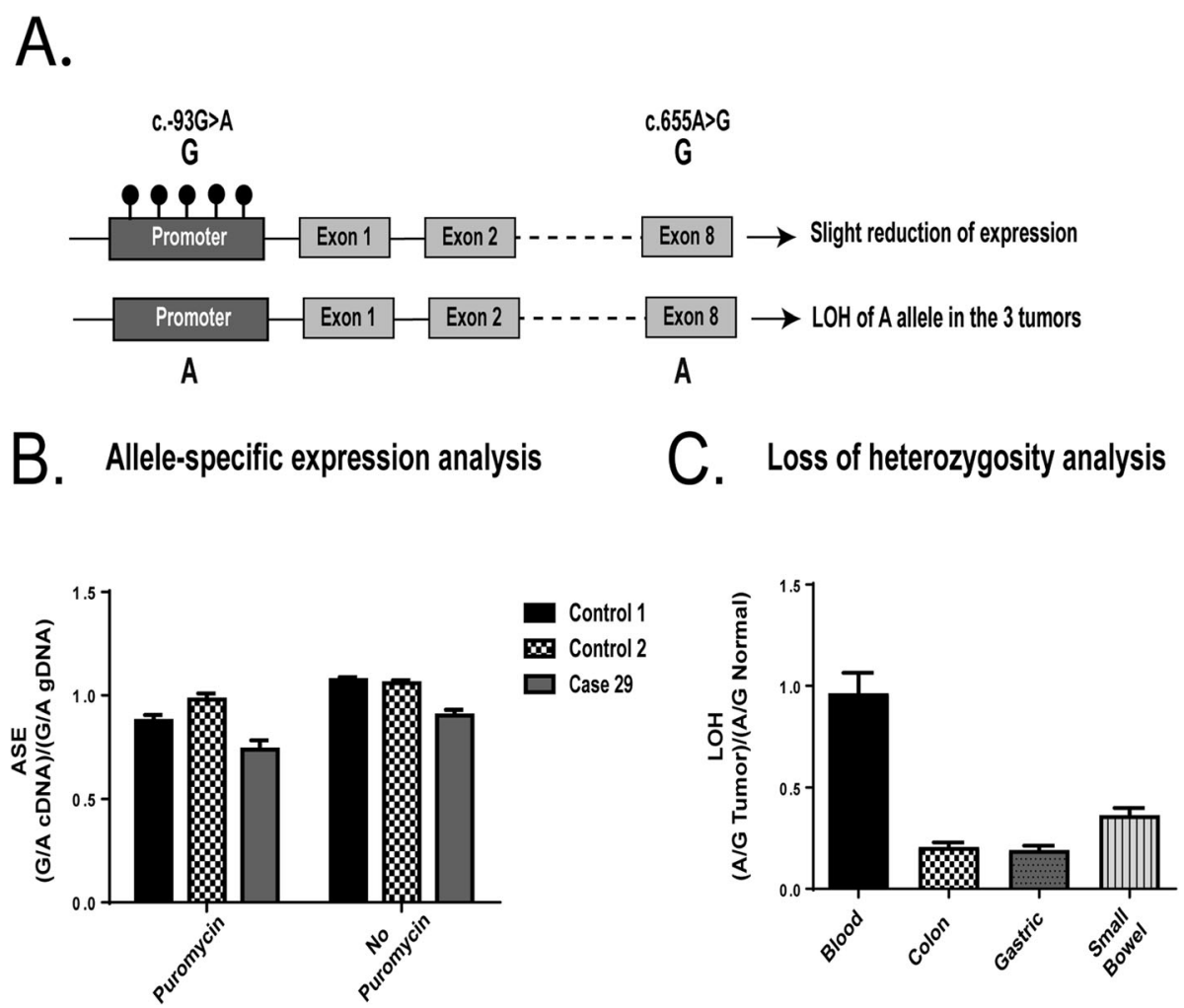

Fig. 3 Analysis of the dose of MLH1 alleles at rs1799977 (c.655A>G). a Schematic representation of the MLH1 epimutation found in case 29. Promoter methylation is associated with the c.-93G allele (in approximately $2 \%$ of cells), located in cis with exonic c.655G allele, which showed subtle reduced transcriptional activity in blood. In concordance, the MLH1 C.655A allele showed LOH in the three tumor lesions developed by the proband. b Allele-specific expression (ASE) analysis at MLH1 c.655G>A in CDNA derived from leukocytes of case 29 and two heterozygous controls. A slightly diminished expression of the c.655G allele was observed. c Loss of heterozygosity (LOH) analysis in tumors from case 29. Allele specific values show loss of the c.655A allele in all three tumors

MCA with clonal bisulfite sequencing and global methylome array analysis confirmed the presence of dense allele-specific methylation spanning the entire EPM2AIP1$M L H 1$ CpG island in a low proportion $(\sim 1 \%)$ of the $M L H 1$ alleles (Additional file 10: Figure S6).

Our MS-MCA approach allowed the robust and highly sensitive detection of $M L H 1$ methylation in blood, whereas no evidence of methylation was detected in controls. In contrast, pyrosequencing and MS-MLPA, widely used in clinical diagnostics for MLH1 methylation detection [12, 14, 23, 24], display a lower analytical sensitivity (5-10\%) than MS-MCA (1\%) [19], potentially overlooking low-level epigenetic mosaicism. Moreover, the background signal observed in pyrosequencing analyses could account for the high proportion $(78 \%)$ of low-level $(<10 \%)$ methylation levels previously reported in healthy controls [17]. Of note, the presence of constitutional methylation was only confirmed in one (case 29) of the five patients showing methylation levels between 1 and $4 \%$ by MSMLPA (Additional file 2: Table S1). Since MS-MLPA is based on methylation-sensitive enzymes, incomplete digestion may account for this apparent inconsistency.
Constitutional epigenetic mosaicism in $M L H 1$ is often observed (reviewed in [9]), in contrast to MMR genetic mosaicism that has been rarely reported [25-27]. Although several cases with low-level epigenetic mosaicism of $M L H 1$ ( $\leq 10 \%$ methylation) have been reported [10, 13-16], to date only seven were validated by other techniques. These reported mosaic cases have shown highly variable clinical phenotypes (Additional file 11: Table S4), being the case identified herein the most expressive. Interestingly, our patient was clinically treated considering the three tumor lesions as non-related primary cancers. Retrospectively, a shared origin of the tumors is highly suggested based on the high percentage of shared mutations [28-30] and the same immunohistochemistry CK20-negative pattern, very rare in CRC [31]. This highlights the clinical complexity of the case.

We did not identify any genetic alteration associated with the epimutated allele in case 29 , including copy number variations, promoter variants within the C-D promoter regions, or other sequencing variants within MLH1 by Sanger or next-generation sequencing using mosaicism pipelines. To date, nine families carrying a 


\section{A. Blood}
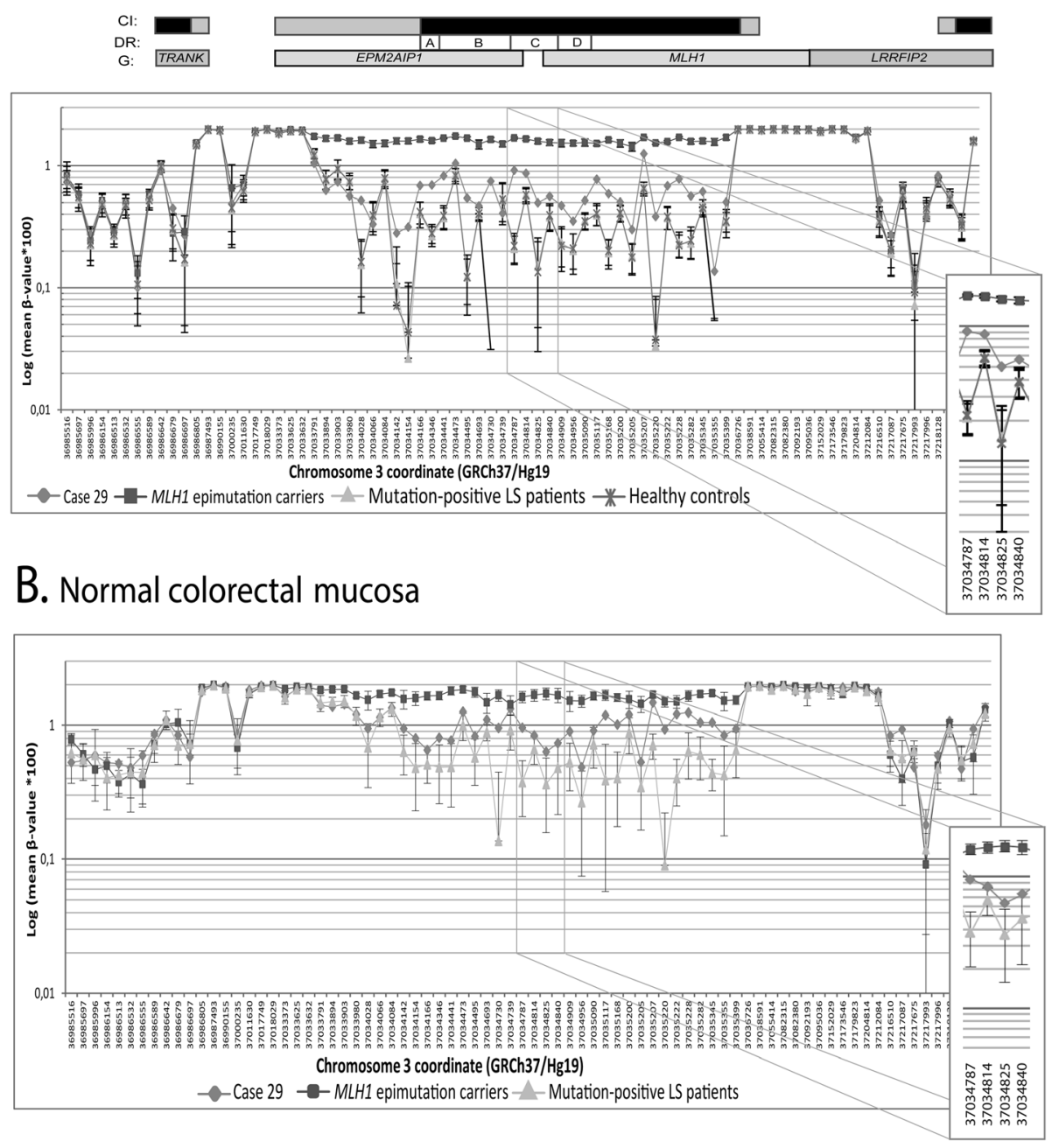

Fig. 4 Representation of the differentially methylated region across the MLH1 locus in blood and colorectal mucosa from case 29, MLH1 epimutation carriers $(n=12)$, Lynch syndrome genetic mutation carriers $(n=61)$, and healthy controls $(n=41)$. Representation of the differentially methylated region across the MLH1 locus in blood DNA (a) and in normal colorectal mucosa (b). $\beta$-values obtained from Infinium 450k Human Methylation array analysis are displayed as a log (mean $\beta$-value $\times 100$ ) against the genomic coordinate for each CpG site interrogated. The relative locations of the CpG sites are not drawn to scale. CpG sites are located between Chr3:36,985,516-37,219,077 coordinates. Above, CpG islands (Cl) are represented as black rectangles and their shores are represented in gray. The location of the Deng regions (DR) A, B, C and D of the MLH1 promoter are indicated by white rectangles. Genes (G) containing the displayed CpG sites are represented as gray rectangles, using the Ensembl GRCh37 database as the reference for gene coordinates

secondary epimutation have been described (reviewed in [8-10]). In one case, the epimutation was present on a low proportion of alleles $(<10 \%)$, associated with the silent variant c.27G>A [10]. Although we cannot completely rule out that genetic alterations have been missed, the lack of family history is also compatible with a de novo primary epimutation. Furthermore, the detection of similar levels of $M L H 1$ methylation in tissues derived from the three embryonic layers suggests that the epimutation arose either during early embryogenesis or as a germline error that was partially erased during early embryogenesis [5].
The phenotypic expressivity of patient 29 contrasts with the subtle functional impact on $M L H 1$ expression, in accordance with the low methylation levels. The possibility of constitutional MMR deficiency in case 29 was formally discarded because of the absence of germline mutations in the promoter and coding regions of $M L H 1$ and the conserved biallelic MLH1 transcription and MLH1 protein expression in normal tissues. Furthermore, no pathogenic alterations in other hereditary cancer genes were detected, although other genetic and/or environmental factors could be playing a role in the observed phenotype. 
In contrast to high-level methylation, the potential contribution of low-level mosaic methylation in blood to cancer risk remains to be properly assessed [6]. The combination of soma-wide allelic methylation and associated transcriptional silencing in a small proportion of cells is consistent with the initiation of carcinogenesis from the $\sim 2 \%$ cells that contained the epimutation. Eventually, the somatic loss of the functional (non-methylated) allele and its clonal expansion would give rise to each of the MLH1-methylated tumors.

Based on the clinical phenotype and the molecular profile, intensive surveillance of metachronous gastrointestinal and gynecological tumors has been recommended to patient 29 [32]. Also, predictive epigenetic testing should be proposed to family members. Unless stable inheritance of hypermethylation could be demonstrated in descendants (as previously reported [14]), the estimation of cancer risk in relatives should be cautious in the absence of an established causal mechanism.

Taking into account the present report, we have identified three bona fide $M L H 1$ epimutation carriers (two previously reported in [18] and one in the present study) among $71(4.2 \%)$ patients with MLH1-methylated CRC and in three of $20(15 \%)$ patients with early onset or multiple tumors (Additional file 1: Figure S1). In all, MLH1 epimutations represent $1 \%$ of all LS cases in our series, including case 29 identified by the use of highly sensitive techniques.

\section{Conclusion}

In summary, we have identified a bona fide case of lowlevel $M L H 1$ epigenetic mosaicism by using highly sensitive MLH1 methylation analysis. Considering the obtained results, we strongly recommend the use of highly sensitive techniques for screening of constitutional methylation in patients diagnosed with early onset and/ or multiple MLH1-methylated tumors. The eventual identification and characterization of additional cases will be critical to ascertain the cancer risks associated with epigenetic mosaicism.

\section{Supplementary information}

Supplementary information accompanies this paper at https://doi.org/10. 1186/s13148-019-0762-6.

Additional file 1. Figure S1. Schematic representation of the origin of the 18 cases included in this study. CRC: colorectal cancer.

Additional file 2: Table S1. Clinical and molecular features of the patients with MLH1 methylated tumors included in this analysis.

Additional file 3. Supplementary Methods.

Additional file 4: Figure S2. MLH1 promoter methylation analysis by Methylation-Specific Melting Curve Analysis (MS-MCA). A) Analytical sensitivity of the promoter $C$ region. The assay displays a sensitivity threshold of $1 \%$. B) Analytical sensitivity of the promoter $D$ region. The assay shows sensitivity around $10 \%$. C) Methylation analysis in blood from 10 healthy controls for the MLH1 C-region. All of them show the same melting curve pattern as the unmethylated control sample, indicating absence of methylation in healthy controls. D) Methylation analysis in blood from 18 patients harboring $\mathrm{MLH1}$ methylated tumors for the promoter $\mathrm{C}$ region. Only case 29 displays low levels of methylation (around 1\%). E) Methylation analysis in tumor and normal gastrointestinal tissues of case 29. F) Methylation analysis in buccal mucosa of case 29. G) Methylation analysis in skin fibroblasts of case 29.

Additional file 5: Figure S3. MLH1 methylation analysis of the promoter C-region and intron 1 by pyrosequencing. A) and B) Analytical sensitivity analysis for the detection of methylation in MLH1 C-region (A) and intron 1 (B). The detection limits for both regions are $4 \%$ and $5 \%$ respectively, enabling the detection of positive samples as those with methylation values greater than 4 or $5 \%$. C) and D) Methylation analysis in blood from case 29 and healthy controls $(n=10-20)$ for the MLH1 Cregion (C) and intron 1 (D). E) and F) Methylation analysis in normal colorectal mucosa from case 29 and Lynch patients $(n=4)$ for MLH1 C-region (E) and intron 1 (F). G) and $\mathbf{H}$ ) Methylation analysis in normal small bowel mucosa and gastric mucosa from case 29 for MLH1 C-region (G) and intron $1(\mathrm{H})$. I) and J) Methylation analysis in normal and gastrointestinal tumor tissues in case 29 for MLHI C-region (I) and intron 1 (J).

Additional file 6: Figure S4. Immunohistochemical characterization of gastrointestinal tumor lesions from patient 29. All tumor lesions are welldifferentiated adenocarcinomas with a variable but not predominant mucinous component. All of them present loss of expression of the cytokeratin markers CK7 and CK20. The transcription factor CDX2 shows strong and diffused positive staining. The expression of the membrane-bound proteins MUC1 and MUC5 is also positive in all tumors but with diffused and lower intensity staining, whereas the MUC2 shows intense, focal and heterogeneous expression. According to this characterization, the three tumors show the same immunohistochemical staining pattern. Objective magnification is 20X for all images. HE, hematoxylin-eosin.

Additional file 7: Table S2. MLH1 methylation assessed by MS-MLPA in samples from case 29.

Additional file 8: Table S3. Variants identified in the mutational analysis of hereditary cancer genes in case 29 .

Additional file 9: Figure S5. Analysis of structural aberrations in case 29. A) Genome-wide SNP array profiling of blood DNA from case 29 is shown as Circos plots. Circos plot was divided into three concentric circles. Chromosomes are represented at the external circle with their centromeres painted in red. In the middle circle, external allelic peaks mark homozygous SNPs and internal allelic peaks heterozygous ones. Internal circle tracks log2 copy number lane: middle points indicate diploid genomic material; upper points, gains of genomic material and lower points, losses. Patient 29 displayed a diploid pattern throughout her genome without signs of loss-of-heterozygosity. B) CNV analysis in the MLH1 region of patient 29 by custom CGH array. Genes located in the analyzed region are represented at the bottom of the figure. Probes are displayed as green dots in a log2 graph. Gains and losses of genetic material are considered when more than five consecutive probes reach values of 2 or -2 , respectively. No CNV abnormalities were identified.

Additional file 10: Figure S6. Schematic representation of the methodological strategy and summary of the obtained results.

Additional file 11: Table S4. Reported patients with MLH1 epigenetic mosaicism at low proportion ( $\leq 10 \%)$. $\left(^{*}\right)$ According to the obtained results the three tumor lesions were clonally related.

Additional file 12: Table S5. A. Primers and conditions. B. Localization of the probes and regions analyzed in the study of MLH1 methylation. The EMP2AIP1-MLH1 CpG island (colored in dark purple) encompass the MLH1 promoter and intron 1.

\section{Abbreviations}

CRC: Colorectal cancer; LOH: Loss of heterozygosity; LS: Lynch syndrome; MMR: Mismatch repair; MS-MCA : Methylation-specific melting curve analysis; MS-MLPA: Methylation-specific multiplex ligation-dependent probe amplification; PBL: Peripheral blood leukocytes; SNuPE: Single-nucleotide primer extension; WGA: Whole Genome Amplification 


\section{Acknowledgements}

We thank the participating patients and families and all the members of the Units of Genetic Counseling and Genetic Diagnostic the Hereditary Cancer Program of the Catalan Institute of Oncology (ICO-IDIBELL). We thank Juana Fernández and Mar Varela for technical assistance.

\section{Authors' contributions}

$\mathrm{ED}, \mathrm{JCH}, \mathrm{GVP}, \mathrm{MP}$, and $\mathrm{GC}$ conceived the project and designed the study. ED, $J C H, A V, E D, A F, A l, G M, G O, C E, V P, H I U, J L S, B Q$, and JB contributed to the data collection. $\mathrm{ED}, \mathrm{JCH}, \mathrm{GVP}, \mathrm{JV}, \mathrm{AF}, \mathrm{FM}, \mathrm{CL}, \mathrm{BQ}, \mathrm{JB}, \mathrm{MH}, \mathrm{MP}$, and $\mathrm{GC}$ carried out the data analysis and its interpretation. $\mathrm{ED}, \mathrm{JCH}, \mathrm{MP}$, and GC wrote the manuscript with input from the rest of authors. Finally, all authors contributed to the critical revision of the manuscript and approved the final version to be published.

\section{Funding}

This work was funded by the Spanish Ministry of Economy and Competitiveness, which is part of Agencia Estatal de Investigación (AEI), and co-funded by FEDER funds - a way to build Europe-(grant SAF2012-33636 and SAF2015-68016-R), CIBERONC, the Spanish Association Against Cancer (080253), and the Government of Catalonia (grants 2014SGR338, 2017SGR1282, and PERIS SLT002/16/0037). ED was supported by a grant from the Spanish Ministry of Economy and Competitiveness. The Mexican National Council for Science and Technology (CONACyT) fellowship to GVP. JCH and FM were supported by CIBERONC, and AF was supported by a grant from the Catalonia Health Department (SLT002/16/00409). We thank CERCA Programme / Generalitat de Catalunya for institutional support.

\section{Availability of data and materials}

The data discussed in this publication is accessible through GEO Series accession number GSE131541 and GSE107353 (https://www.ncbi.nlm.nih.gov/geo/query/acc.cgi?acc=GSE131541 and https://www.ncbi.n/m.nih.gov/geo/query/acc.cgi?acc=GSE107353).

\section{Competing interest}

GC and JdV have received personal fees from VCN Biosciences and AstraZeneca, respectively, outside the submitted work. There are no other relationships or activities that could appear to have influenced the submitted work. All other authors declare that they have no competing interest.

\section{Ethics approval and consent to participate}

This study was approved by the Ethics Committee of the Institut d'Investigació Biomèdica de Bellvitge (IDIBELL). Specimens were obtained after the patients had provided written informed consent.

\section{Consent for publication}

We obtained written informed consent from the patient to publish patient's clinical and molecular information.

\section{Author details}

${ }^{1}$ Hereditary Cancer Program, Catalan Institute of Oncology, Institut d'Investigació Biomèdica de Bellvitge (IDIBELL), ONCOBELL Program, Av. Gran Via de l'Hospitalet, 199-203, 08908 L' Hospitalet de Llobregat, Barcelona, Spain. ${ }^{2}$ Department of General and Digestive Surgery, Dr Josep Trueta University Hospital, Girona, Spain. ${ }^{3}$ Hereditary Cancer Program, Catalan Institute of Oncology, Institut d'Investigació Biomèdica de Girona (IDIBGI), Girona, Spain. ${ }^{4}$ Pathology Department, Dr Josep Trueta University Hospital, Girona, Spain. ${ }^{5}$ Pathology Department, Clínica Girona, Girona, Spain. ${ }^{6}$ Department of Gastroenterology, Dr Josep Trueta University Hospital, Girona, Spain. ${ }^{7}$ Hereditary Cancer Program Valencian Region, Molecular Genetics Laboratory, Elche University Hospital, Elche, Alicante, Spain. ${ }^{8}$ Department of Biomedical Sciences, Cedars-Sinai Medical Center, CA, Los Angeles, USA. ${ }^{9}$ Centro de Investigación Biomédica en Red de Cáncer (CIBERONC), Madrid, Spain. ${ }^{10}$ Department of Medical Oncology, Catalan Institute of Oncology, Girona, Spain. ${ }^{11}$ Department of Medical Sciences Department, School of Medicine, University of Girona, Girona, Spain.
Received: 23 May 2019 Accepted: 9 October 2019

Published online: 28 November 2019

\section{References}

1. Hansmann T, Pliushch G, Leubner M, Kroll P, Endt D, Gehrig A, et al. Constitutive promoter methylation of BRCA1 and RAD51C in patients with familial ovarian cancer and early-onset sporadic breast cancer. Hum Mol Genet. 2012;21(21):4669-79.

2. Bennett KL, Mester J, Eng C. Germline epigenetic regulation of KILLIN in Cowden and Cowden-like syndrome. JAMA. 2010;304(24):2724-31.

3. Raval A, Tanner SM, Byrd JC, Angerman EB, Perko JD, Chen SS, et al. Downregulation of death-associated protein kinase 1 (DAPK1) in chronic lymphocytic leukemia. Cell. 2007;129(5):879-90.

4. Gelli E, Pinto AM, Somma S, Imperatore V, Cannone MG, Hadjistilianou T, et al. Evidence of predisposing epimutation in retinoblastoma. Hum Mutat. 2019;40(2):201-6.

5. Hitchins MP. Constitutional epimutation as a mechanism for cancer causality and heritability? Nat Rev Cancer. 2015;15(10):625-34.

6. Lønning PE, Eikesdal HP, Løes IM, Knappskog S. Constitutional Mosaic Epimutations - a hidden cause of cancer? Cell Stress. 2019;3(4):118-35.

7. Lynch HT, Snyder CL, Shaw TG, Heinen CD, Hitchins MP. Milestones of Lynch syndrome: 1895-2015. Nat Rev Cancer. 2015;15(3):181-94.

8. Morak M, Ibisler A, Keller G, Jessen E, Laner A, Gonzales-Fassrainer D, et al. Comprehensive analysis of the MLH1 promoter region in 480 patients with colorectal cancer and 1150 controls reveals new variants including one with a heritable constitutional MLH1 epimutation. J Med Genet. 2018:55(4):240-8.

9. Dámaso E, Castillejo A, Arias MDM, Canet-Hermida J, Navarro M, Del Valle J, et al. Primary constitutional MLH1 epimutations: a focal epigenetic event. $\mathrm{Br}$ J Cancer. 2018;119(8):978-87.

10. Leclerc J, Flament C, Lovecchio T, Delattre L, Ait Yahya E, Baert-Desurmont $\mathrm{S}$, et al. Diversity of genetic events associated with MLH1 promoter methylation in Lynch syndrome families with heritable constitutional epimutation. Genet Med. 2018;20(12):1589-99.

11. Barrington $\mathrm{DA}$, Champion ML, Boitano TKL, Walters-Haygood CL, Farmer MB, Alvarez RD, et al. Characteristics of African American women at high-risk for ovarian cancer in the southeast: results from a gynecologic cancer risk assessment clinic. Gynecol Oncol. 2018;149(2):337-40.

12. Pinto D, Pinto C, Guerra J, Pinheiro M, Santos R, Vedeld HM, et al. Contribution of MLH1 constitutional methylation for Lynch syndrome diagnosis in patients with tumor MLH1 downregulation. Cancer Med. 2018; 7(2):433-44.

13. Hitchins MP, Owens SE, Kwok CT, Godsmark G, Algar UF, Ramesar RS. Identification of new cases of early-onset colorectal cancer with an MLH1 epimutation in an ethnically diverse South African cohort. Clin Genet. 2011; 80(5):428-34.

14. Sloane MA, Nunez AC, Packham D, Kwok CT, Suthers G, Hesson LB, et al. Mosaic epigenetic inheritance as a cause of early-onset colorectal cancer. JAMA Oncol. 2015;1(7):953-7.

15. Ward RL, Dobbins T, Lindor NM, Rapkins RW, Hitchins MP. Identification of constitutional MLH1 epimutations and promoter variants in colorectal cancer patients from the Colon Cancer Family Registry. Genet Med. 2013; 15(1):25-35.

16. Wu PY, Zhang Z, Wang JM, Guo WW, Xiao N, He Q, et al. Germline promoter hypermethylation of tumor suppressor genes in gastric cancer. World J Gastroenterol. 2012;18(1):70-8.

17. Auclair J, Vaissière T, Desseigne F, Lasset $C$, Bonadona V, Giraud $S$, et al. Intensity-dependent constitutional MLH1 promoter methylation leads to early onset of colorectal cancer by affecting both alleles. Genes Chromosomes Cancer. 2011;50(3):178-85.

18. Pineda $M$, Mur $P$, Iniesta $M D$, Borràs $E$, Campos $O$, Vargas $G$, et al. MLH1 methylation screening is effective in identifying epimutation carriers. Eur J Hum Genet. 2012;20(12):1256-64.

19. Gausachs M, Mur P, Corral J, Pineda M, González S, Benito L, et al. MLH1 promoter hypermethylation in the analytical algorithm of Lynch syndrome: a cost-effectiveness study. Eur J Hum Genet. 2012;20(7):762-8.

20. Ostrovnaya I, Seshan VE, Olshen AB, Begg CB. Clonality: an R package for testing clonal relatedness of two tumors from the same patient based on their genomic profiles. Bioinformatics. 2011;27(12):1698-9.

21. Network CGA. Comprehensive molecular characterization of human colon and rectal cancer. Nature. 2012;487(7407):330-7. 
22. Kwok CT, Vogelaar IP, van Zelst-Stams WA, Mensenkamp AR, Ligtenberg MJ, Rapkins RW, et al. The MLH1 C.-27C >A and c.85G>T variants are linked to dominantly inherited MLH1 epimutation and are borne on a European ancestral haplotype. Eur J Hum Genet. 2014;22(5):617-24.

23. Castillejo A, Hernández-Illán E, Rodriguez-Soler M, Pérez-Carbonell L, Egoavil C, Barberá VM, et al. Prevalence of MLH1 constitutional epimutations as a cause of Lynch syndrome in unselected versus selected consecutive series of patients with colorectal cancer. J Med Genet. 2015;52(7):498-502.

24. Crucianelli F, Tricarico R, Turchetti D, Gorelli G, Gensini F, Sestini R, et al. MLH1 constitutional and somatic methylation in patients with MLH1 negative tumors fulfilling the revised Bethesda criteria. Epigenetics. 2014; 9(10):1431-8.

25. Pastrello C, Fornasarig M, Pin E, Berto E, Pivetta B, Viel A. Somatic mosaicism in a patient with Lynch syndrome. Am J Med Genet A. 2009;149A(2):212-5.

26. Sourrouille I, Coulet F, Lefevre JH, Colas C, Eyries M, Surcek M, et al. Somatic mosaicism and double somatic hits can lead to MSI colorectal tumors. Familial Cancer. 2013;12(1):27-33.

27. Geurts-Giele WR, Rosenberg EH, Rens AV, Leerdam MEV, Dinjens WN, Bleeker FE. Somatic mosaicism by a de novo MLH1 mutation as a cause of Lynch syndrome. Mol Genet Genomic Med. 2019;7(7):e00699.

28. Schultheis AM, Ng CK, De Filippo MR, Piscuoglio S, Macedo GS, Gatius S, et al. Massively parallel sequencing-based clonality analysis of synchronous endometrioid endometrial and ovarian carcinomas. J Natl Cancer Inst. 2016; 108(6):djv427.

29. Vakiani E, Janakiraman M, Shen R, Sinha R, Zeng Z, Shia J, et al. Comparative genomic analysis of primary versus metastatic colorectal carcinomas. J Clin Oncol. 2012;30(24):2956-62

30. Mauguen A, Seshan VE, Ostrovnaya I, Begg CB. Estimating the probability of clonal relatedness of pairs of tumors in cancer patients. Biometrics. 2018; 74(1):321-30.

31. Chu PG, Weiss L. Modern immunohistochemistry. 2nd. edition. New York: Cambridge University Press; 2014.

32. Giardiello FM, Allen Jl, Axilbund JE, Boland CR, Burke CA, Burt RW, et al. Guidelines on genetic evaluation and management of Lynch syndrome: a consensus statement by the US Multi-society Task Force on colorectal cancer. Am J Gastroenterol. 2014;109(8):1159-79.

\section{Publisher's Note}

Springer Nature remains neutral with regard to jurisdictional claims in published maps and institutional affiliations.

Ready to submit your research? Choose BMC and benefit from:

- fast, convenient online submission

- thorough peer review by experienced researchers in your field

- rapid publication on acceptance

- support for research data, including large and complex data types

- gold Open Access which fosters wider collaboration and increased citations

- maximum visibility for your research: over $100 \mathrm{M}$ website views per year

At $\mathrm{BMC}$, research is always in progress.

Learn more biomedcentral.com/submissions 